\title{
The novel feature based inspection technique that can detect defects that can affect the deterioration of the electrical properties of semiconductor devices
}

\author{
Sungyoon Ryu ${ }^{1}$, Younghoon Sohn ${ }^{2}$ and Yusin Yang ${ }^{2}$ \\ ${ }^{1}$ Samsung electronics, Republic of Korea, ${ }^{2}$ Samsung electronics, Seoul-t'ukpyolsi, Republic of Korea
}

\section{INTRODUCTION}

As the semiconductor industry has been moving toward increasing the number of transistors on integrated circuit, developing the powerful defect inspection system for the complicated nano structures becomes significantly important. Although the advanced manufacturing process enables us to make logic and memory devices include sub-20nm structures, the higher device yield will be only achieved by utilizing precise metrology and inspection tools. The defect inspection technique can be divided into two categories; an optical method and an electron beam method. Compared to the e-beam solution, the optical inspection technique is known to provide much higher throughput at the cost of sacrificing resolution. Although the current optical solution together with sophisticated algorithms will be able to detect defects those were much smaller than physical limitation of optical imaging, imaging sub-20 nm defects with high sensitivity will never be easy to achieve. To overcome this, we present the completely new inspection method that able to detect process-variation-induced defects those were not fully detected by the traditional inspection method.

\section{OPTICAL INSPECTION PRINCIPLES}

While the wafer can be quickly scanned along the horizontal direction, the two images of neighboring dies (or cells) were simultaneously compared to identify the existence of the defects, where one becomes the inspection image and another one becomes the reference image. The highly precise alignment, subtraction, and filtering algorithms are essential. All the defect data including defect number (arbitrarily assigned), positions, and their size were stored in the database to generate and display all the defects in the wafer map. The defect can be identified when the strength of signal exceeds certain threshold value the user defined. The detection SNR of defects significantly varies depending on their size and shape. Our main interest is to detect challenging defects those were typically very small over the complicated 2-D structures, resulting in the poor SNR.

Researchers has been putting significant efforts to further improve the detection capability of such challenging defects by adapting high NA optics and reducing the wavelength, but technology advancement relying on this kind of traditional HW development approach now becomes very limited. Under the given HW limitation, the only remaining approach will be developing the new algorithm. To realize that, KLA-Tencor recently proposed the new algorithm called PINPOINT to reduce the pattern noise, but this can be only used for the limited applications [2]. In this paper, we propose the new method named feature based inspection (FBI) to boost imaging capability of detecting challenging defects by taking into account the additional new process-sensitive-feature without improving HW performance.

\section{FEATURE BASED INPECTION (FBI)}

Changing the wafer manufacturing process will directly cause the pattern profile variation. The optimal inspecting conditions such as wavelength, apertures, polarization, etc., must be selected to provide the 
greatest correlation between process variation and the strength of defect signal represented by the certain feature, where we utilize that particular feature to inspect defects and the method works in the following order.

(1) Prepare the wafer exposed by different focus dose values, then inspect that wafer with all possible optical conditions (wavelength, aperture, polarizer, focus, magnification, etc.) in the BF tool

(2) Analyze the correlation between specific feature values (Magnitude, brightness, Defect size, etc) and process-variation induced structural change

(3) Check the strength of defect signal measured for all optical conditions

(4) Define the optimal inspection conditions, providing the highest correlation measured in step (2) together with the highest defect signals measured in step (3)

(5) Generate the golden reference image for the structures in the die where the region was exposed with the best process condition

(6) Obtain the signal difference between the golden reference image in (5) and inspection images for the optimal condition defined in (4), where the defect signal can be amplified by adding feature offset

(7) Rebuild the defect map based on the most sensitive features determined in (2).

By utilizing FBI technique, defect signal can be significantly enhanced due to the addition of additional feature offset values, resulting in the improved detection capability of the challenging defects.

\section{Experimental VALIDATION}

To validate the FBI method, we first evaluate identifying gate bit line (GBL) necking failure happened in DRAM manufacturing process, where such defects were very challenging to detect by using the conventional inspection method in the BF tool. Throughout the FBI sequence mentioned in the previous section, we can obtain the correlation between the magnitude value (the main feature) and GBL poly CD for various different optical conditions, that enables us to select the optimal inspection condition. Simultaneously, we can validate the optimal inspection condition through RCWA based optimal simulation, where the best condition provide the highest defect sensitivity.

\section{CONCLUSION}

We present a novel FBI method that enables us to boost imaging capability of detecting challenging defects those were not effectively captured in the conventional inspection systems. We validate this approach to detect randomly occurred GBL necking defects.

References

[1] K.W. Tobin submission for: V. Sankaran, C.M. Weber, K.W. Tobin "Inspection in Semiconductor Manufacturing", Webster's Encyclopedia of Electrical and Electronic Engineering, vol. 10, pp. 242-262, Wiley \& Sons, NY, NY, 1999.

[2] A. Blauberg, et all, "Understanding process and design systematics: case study on monitoring strategy and understanding root cause of fin defectively, ASMC, 2017 